\title{
A multi-scale modeling approach to predict the impact of clinical interventions on femoral growth in children with cerebral palsy
}

Hans Kainz ${ }^{1}$, Mariska Wesseling ${ }^{1}$, Sanne Vancleef ${ }^{1}$, Fernando Perez Boerema ${ }^{1}$, S Mahsa Sadeghian ${ }^{2}$, Friedl De Groote $^{1}$, Kaat Desloovere ${ }^{1}$, Jose Manuel Garcia Aznar ${ }^{3}$, Sandra Shefelbine ${ }^{2}$, Ilse Jonkers ${ }^{1}$

\author{
${ }^{1} \mathrm{KU}$ Leuven, Leuven, Belgium \\ ${ }^{2}$ Northeastern University, Boston, USA \\ ${ }^{3}$ University of Zaragoza, Zaragoza, Spain
}

\section{Introduction}

Many children with cerebral palsy (CP) are born with typical bones but develop bone deformities during growth due to abnormal bony loadings caused by their pathological gait pattern. While Botulinum toxin $A(B T X-A)$ injections are planned to reduce spasticity, their impact on bone growth remains unclear.

\section{Research question}

Do BTX-A injections normalize femoral growth in children with CP?

\section{Methods}

Motion capture data of one typically developing (TD) and three children with spastic diplegic $\mathrm{CP}$, before and after BTX-A treatment, were used for musculoskeletal modeling and dynamic simulations of gait. The CP children walked with apparent equinus (AE), jump gait (JG) and crouch gait (CG). Muscle forces were calculated using an electromyography-constrained approach to take the individual motor control into account. Afterwards, hip joint contact forces (HJCF) were calculated using OpenSim. The calculated muscle and HJCF were used as loading conditions in a finite element (FE) model of the femur, developed from magnetic resonance images of the TD child. For each participant, femoral growth rate was calculated as the osteogenic index, which was based on the minimum hydrostatic stress and maximum octahedral shear stress at the growth plate obtained from the FE analysis using Abaqus. Growth direction was defined based on the maximum absolute principal stress magnitude at each element of the growth plate [1]. Joint kinematics, HJCF and growth trends were compared before and after BTX-A injections.

\section{Results}

After the BTX-A treatment, overall joint kinematics improved for the AE- and CG-child and did not change for the JG-child (Fig.1). Root-mean-square-differences (RMSD) between the CP and TD child's joint kinematics were $8.4^{\circ}, 10.4^{\circ}$ and $10.1^{\circ}$ before and $7.1^{\circ}, 10.4^{\circ}$ and $8.5^{\circ}$ after BTX-A for the AE-, JGand CG-child, respectively.

In the AE-child, BTX-A led to normalized (closer to TD values) HJCF (pre-/post-RMSD:1.2/0.8 body weight (BW)), whereas in the JG-child, an increased deviation (pre-/post-RMSD:0.7/0.9 BW) and in the CG-child no change (pre-/post-RMSD:1.2/1.2 BW) in HJCF were observed.

The osteogenic index was different between the TD child and all CP children (Fig.1). As expected, the TD loading led to decreased femoral anteversion and neck-shaft angle. In CP, the anteversion angle and neck-shaft angle remained unchanged. BTX-A injections had a small impact on the osteogenic index and femoral growth trend in the AE-child and a negligible impact in the JG- and CG-child.

\section{Discussion}


Our TD and pre-BTX-A femoral grow simulation results were in agreement with longitudinal studies based on medical images [2]. Only in the AE child, BTX-A had a positive impact on femoral bone growth. The AE child was the only participant in which HJCF improved after BTX-A. In the future we will investigate if the HJCF can be used as an predictor for femoral bone growth.
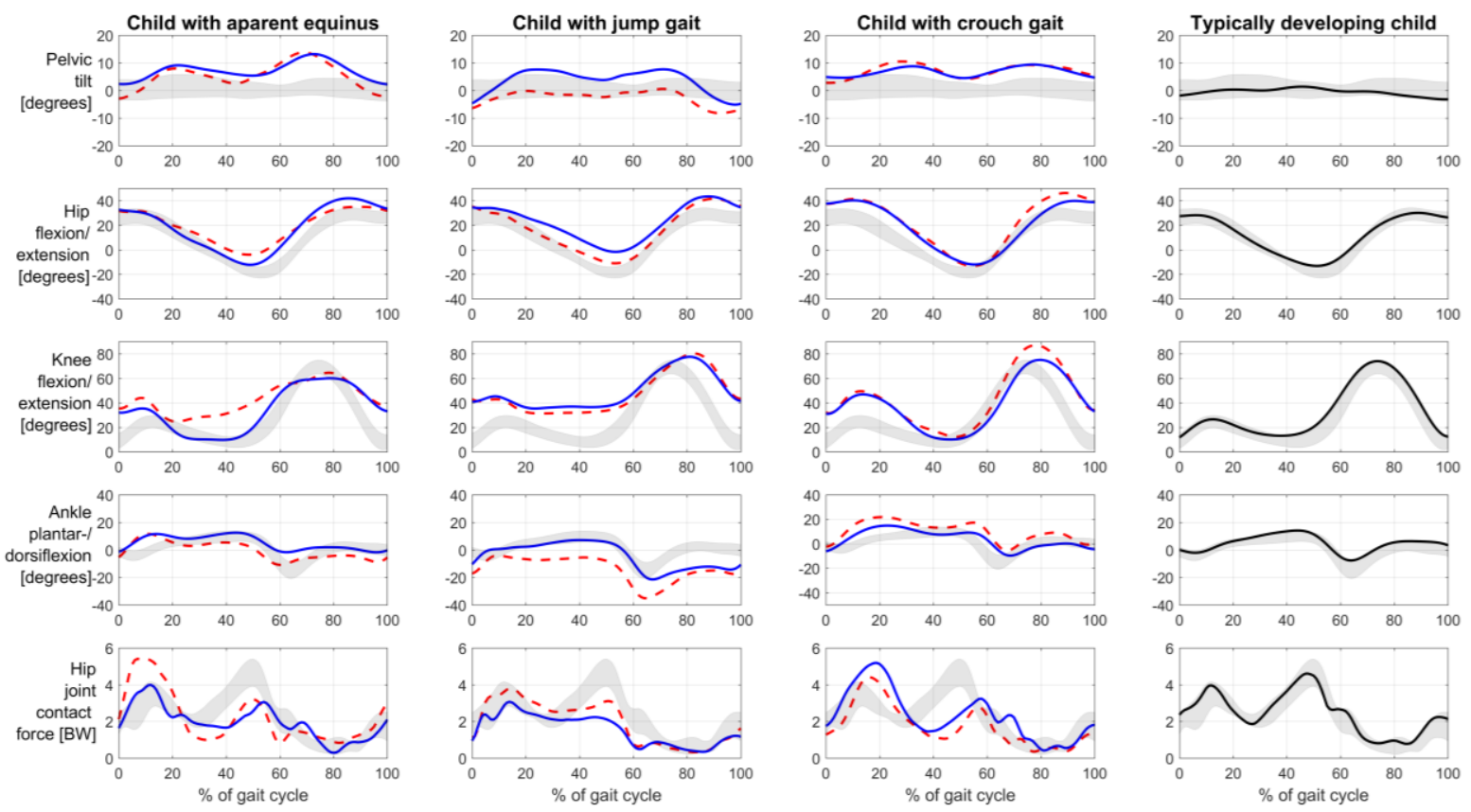

- - - preBTX-A

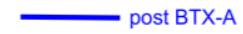

TD reference data
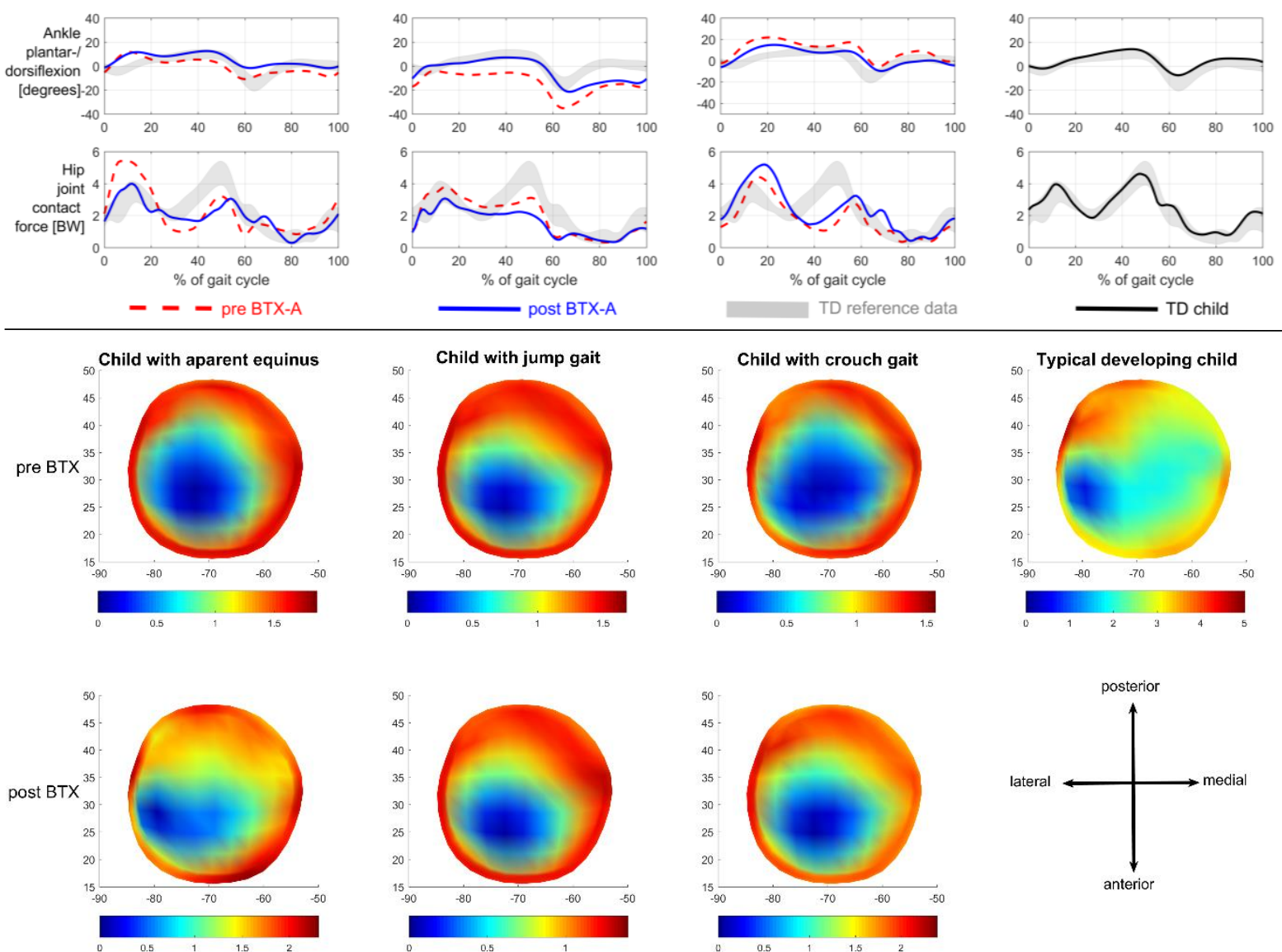

Fig.1. Sagittal plane kinematics (row 1 to 4), HJCF (row 5) and osteogenic index (bottom two rows) for all participants.

\section{References}

[1] Yadav et al. J.Biomech. 49 (2016) 1613-1619.

[2] Bobroff et al. Clin.Orthop.Relat.Res. (1999) 194-204. 
